\title{
A Modified and Improved Method for Detection of Tumor in Brain Cancer
}

\author{
Meenakshi Sharma \\ CSE Department \\ SSCET Badhani, Pathankot, India
}

\author{
Simranjit Singh \\ CSE Department \\ SSCET Badhani, Pathankot, India
}

\begin{abstract}
The precise information of a tumor plays an important role in the treatment of malignant tumors. The manual segmentation of brain tumors from Magnetic Resonance images (MRI) is time consuming task. Processing of MRI images is one of the parts of this field. The detection and extraction of tumor is done from patient's MRI scan images of the brain. The basic concepts of the image processing are some noise removal functions, segmentation and morphological operations. The modified image segmentation and histogram thresh holding techniques were applied on MRI scan images in order to detect brain tumors. In addition, a region prop and skull is used to detect the tumor in the brain. The proposed method can be successfully applied to detect the contour of the tumor and its geometrical dimension. The result of present paper has been very promising.
\end{abstract}

\section{Keywords}

MRI, Histogram, brain tumor detection, tumor identification, segmentation.

\section{INTRODUCTION}

Tumor is generally defined as the abnormal growth of the tissues. Brain tumor is an abnormal mass of tissue in which cells grow and multiply uncontrollably, in brain this mechanism is unchecked by the control normal cells. Brain cancer can be counted among the most deadly and intractable diseases. Brain tumors can be primary or metastatic, and either malignant or benign. A metastatic brain tumor is a cancer that has spread from elsewhere in the body to the brain. It is a kind of brain disorder in which clusters of nerve cells, or neurons, in the brain sometimes signal abnormally. MRI helps in finding the tumor in the brain. MRI is used to produce high quality images of the parts contained in the human body. The MRI imaging is often used when treating brain tumors, ankle, and foot. These images are also known as the high resolution images. With the help of these images one can derive the detailed anatomical information to examine human brain development and discover abnormalities. Many methodologies are used for classifying MRI. These techniques are fuzzy methods, neural networks, atlas methods, knowledge based techniques, shape methods, variation segmentation. The MRI consists of T1 weighted, T2 weighted and proton density weighted images. These are processed by a system which integrates fuzzy based technique with multispectral analysis. Imaging plays a central role in the diagnosis and treatment planning of brain tumor. For this purpose many methods are use. The MR imaging method is the best due to its higher resolution.

The anatomy of brain states that the patients with the single brain metastasis may benefit from cytoreductive surgery in the combination with whole brain radiotherapy in terms of prolonged functionally independent survival though overall survival has not been shown to improve. Meningioma's represent the other end of the scale as these tumors may undergo gross total resection with high rates of the long term survival. It is used in order to obtain the relevant tissue for histological investigations remain the primary diagnostic modality. In case of brain abscesses, puncture and culturing of sampled material provides a microbiological diagnosis. Stereotactic biopsies used the frame based systems. These systems are well documented in terms of precision, high diagnostic yield and low rate of complications. The stereotactic computers are applied in neurosurgery. These systems are helpful tool in pre operative planning. A stereotactic computer has been used instead of a frame for various intracranial procedures. For the better detection off the brain tumor a skull mounted guide system was developed. This system is coupled with a stereotactic computer and it permits the puncture of intracranial mass lesions. The morphologic investigations of tumor tissue remain a gold standard for diagnosing brain tumors. A final diagnosis is commonly based upon post operative histology evaluation of the paraffin embedded material.

\section{RELATED WORK}

Minakshi Sharma et.al [9], in this paper author discussed about the detection and segmentation of brain tumor, because it provides anatomical information of normal and abnormal tissues. It helps in treatment planning and patient follow up. There are number of techniques for image segmentation. In this paper ANFIS (Artificial Neural Network Fuzzy Inference System) technique is used for image classification and then compares the results with FCM (Fuzzy C means) and K-NN (K-nearest neighbor). ANFIS includes benefits of both ANN and the fuzzy logic systems.

D. Jayadevappa et.al[10], discuss about the Medical Image segmentation. It generally deals with segmentation of tumor in CT and MR images for improved quality in medical diagnosis. The geometric vector flow (GVF) enhances the concave object extraction capability. It also suffers from high computational requirement and sensitiveness to noise. In this paper author combines the watershed algorithm with GVF snake model.

Mukesh Kumar et.al [11] discuss that the detection and segmentation of brain tumor accurately is a challenging task in MRI. The automatic seed finding methods may suffer with the problem if there is no growth of tumor and any small white part is there. When the edges of tumor are not sharp then the segmentation results are not accurate. This may be happened due to initial stage of the tumors automatically is proposed. Segmentation is a process of identifying

K.Selvanayaki et.al[12]: the authors discuss about the automatic detection of brain tumor through Magnetic Resonance Image (MRI) used in different stages of Computer Aided Detection System (CAD). In this Image Acquisition, preprocessing and enhancement, segmentation steps are compared. In Preprocessing and Enhancement stage, medical 
image is converted into standard format with contrast manipulation.

In 2012,Manoj K Kowar et.al [16] discuss about the segmentation issues in MRI. As the manual segmentation of brain tumors from Magnetic Resonance images is a challenging and time consuming task. In this paper author discuss a novel technique for the detection of tumor in brain using segmentation and histogram thresh holding.

In 2012, Kailash Sinha et.al [17] discussed about the detection of brain tumor using the segmentation. The segmentation is used to find the various tissues presented in the brain like white matter, gray matter and tumor. The clustering algorithms are used to classify the image layer by layer. The lowest level weight vector is found by the abstraction level. By the use of this technique the higher level of tumor pixel achieved.

In 2012, Dina Aboul Dahab et.al [18] discuss that the detection techniques of the brain tumor using PNN. The modified image segmentation techniques were applied on MRI scan images in order to detect the brain tumors. A modified Probabilistic Neural Network (PNN) model is based on learning vector quantization (LVQ) with image and data analysis. The assessment of the modified PNN classifier performance is measured in terms of the training performance, classification accuracies and computational time.

In 2012, Garima Garg Sonia Juneja [19] discuss that the image processing is any type of signal processing technique. In the image processing any abnormal image of brain tumor is taken. An extracted portion of tumor can be obtained by applying genetic algorithm with fuzzy clustering means method. FCM is superior over different clustering approaches. The combined approach is used to improve segmentation efficiency.

In 2013, Meghana Nagori et.al [20]: in this paper author discuss about the metabolite values. As the brain tumor patients are increasing day by day. Metabolites values are used to detect the brain tumor. Some of the metabolites values are NAA, Creatine, Choline and $\mathrm{Cr} 2$. NAA ratio is used to detect the tumor in the brain. As it decided the tumor type so weights are assigned to each metabolite while clustering.

In 2013,Dr.N. Nandha Gopal [21] discussed about the Magnetic Resonance Imaging. MRI plays an important role in Brain Tumor diagnosisation in advanced stages. It is a form of medical imaging using nuclear magnetic resonance of protons in the body. The segmentation process is used to extract suspicious region from complex medical images.

In 2013, Vivek Angoth et.al [22], discuss about diagnosis of the tumor. As the accurate detection of size and location of brain tumor plays a vital role in the diagnosis of tumor. In this paper, an efficient wavelet based algorithm used for tumor detection. It utilizes the complementary and redundant information from the computed tomography (CT) image and Magnetic Resonance Imaging (MRI) images.

\section{PROPOSED WORK}

Ultra sound brain tumor images consist of speckle noise. The main problem in the current work is to purpose a system for identifying the brain cancer tumor from ultra sound images. Initially the segmentation will be done for detecting the tumor. The speckle noise will be suppressed the next phase features will extracted out of the segmented image. The feature extracted of the image will represent its properties and on the analysis of values of the texture feature parameters The ultrasound image will be classified as benign and malignant brain cancer tumor. Here to solve this problem, the region based and the skull method is used for the detection of the tumor. They have several advantages, but line and edge information in computer vision systems are also important.

\subsection{Idle State}

it is the intial stage. In this a object is taken. The object is defined in the different pixels.

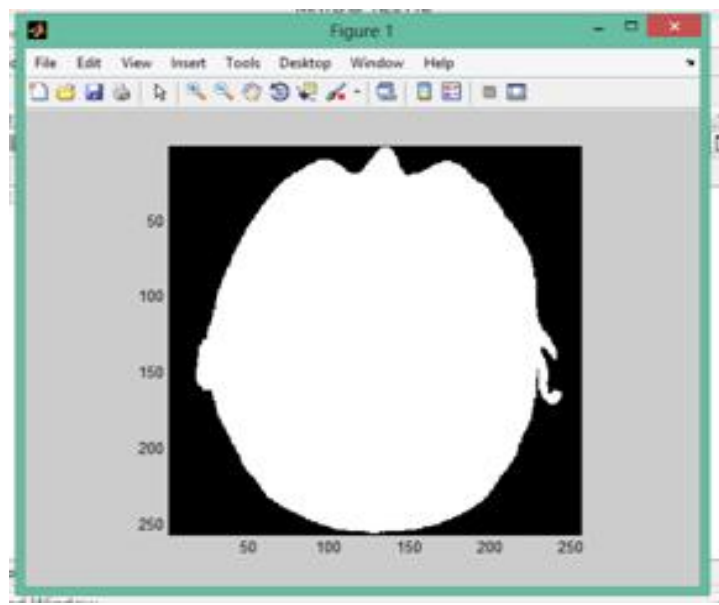

Fig 1: Image reconstruct with only object

This is simple figure which reconstruct with only object figure, because to detect brain tumor the thing is needed is only scanned object. So firstly we reconstruct object only. The proposed method is a combination of region and skull information. It detects the tumor part from scanned image and draws a histogram on it.

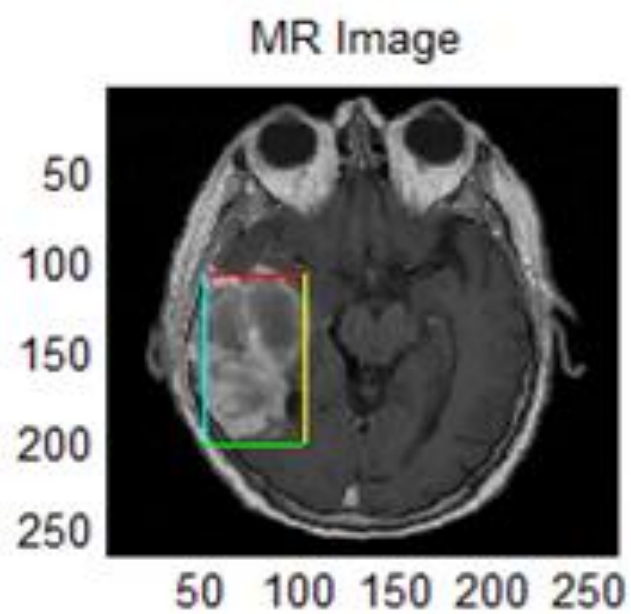

Fig 2: Magnetic Resonance Imaging (MRI)

In MRI descriptors function used which find the region or place where the problem occur, then after that region props function draw the region on that particular object in image In the brain tumor detection, MRI Plays a vital role. In our purposed approach MRI is used after that EDAMAS is used, which helps to find out the swelling of the soft tissues. After that we use the descriptor, region growing and the masking algorithms. 


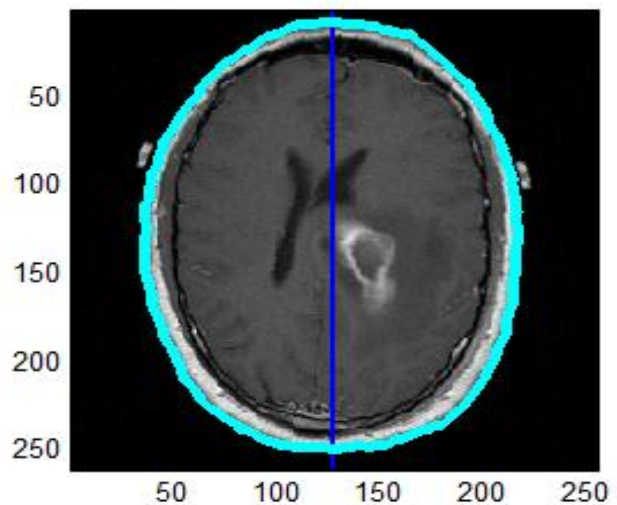

Fig 3: Image divided into 2 segments

Draw the boundary of image using region growing and divided into 2 segments. It helpful in finding the position of the tumor.

Draw the boundary of image using region growing and divided into 2 segments.

\section{Vertical Scanning:}

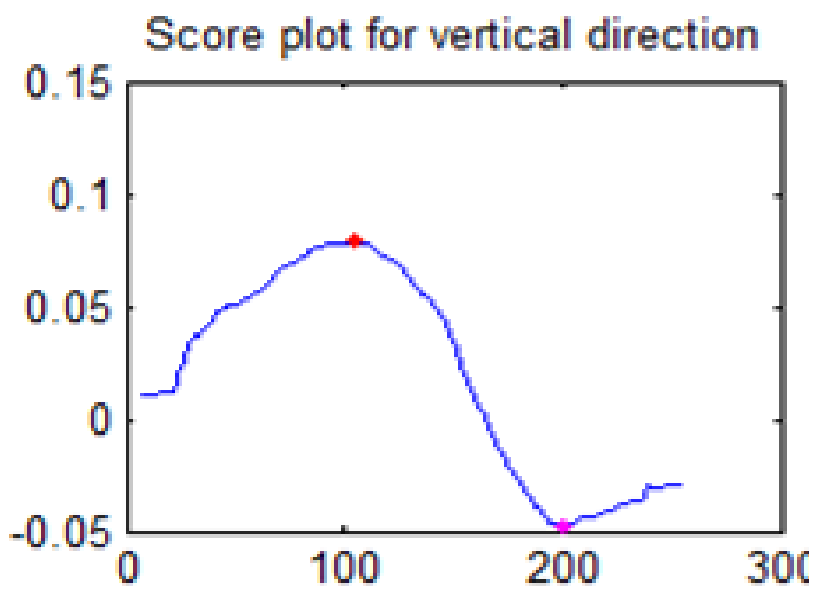

Fig 4: Vertical scanning

In Vertical Scanning it shows the size of effected region, two points on line shows the size of tumor.

\section{Horizontal Scanning:}

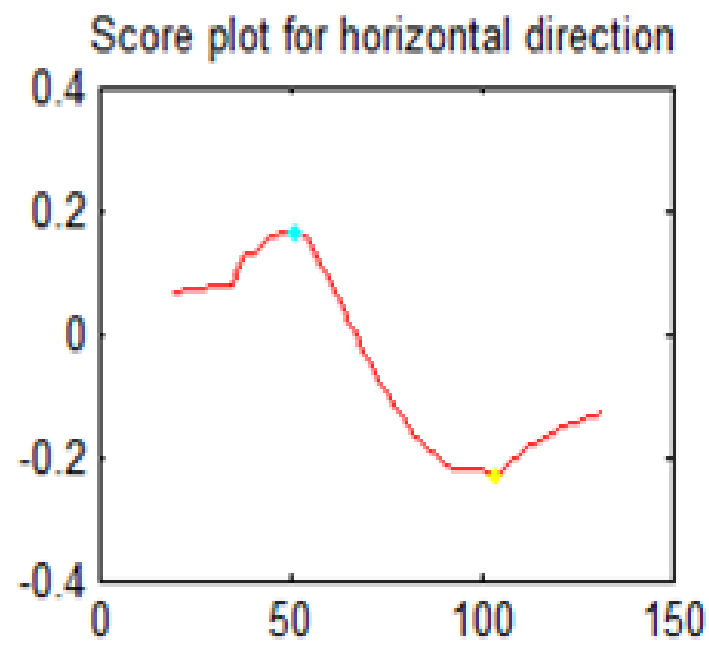

Fig 5: Horizontal scanning

In Horizontal Scanning it shows the size of effected region, two points on line shows the size of tumor.

\section{ALGORITHM:}

Step1: Read image using * function which have properties to select any image

Step2: After that detecting the skull

Step3: Region props function used which draw the region for an image just 2-3 sec than after that we display that MR image using display function, the image show in particular subplot in which we given the axis value using axis function.

Step4: After that we create the mask for in which we have further steps.

- left image, we call this original/test image

- $\quad$ mask for original image

- reference image, here it is the right side

- $\quad$ mask for reference image

Step5: When all result will declare means final o/p show we create two types scanning vertical and horizontal this can be done using round function.

Step6: After that we made histogram for particular figure which we want, the use age of histogram is that it is block is a Signal Processing Block set block. Draw the block for particular detection in which the process in the descriptors is -

- $\quad$ compute the image gradients

- $\quad$ round off

- Within the big square, select the pixels with the circle and put into the histogram. no need to do rotation which is very expensive

Step7: The main function in which we find the max and min score function for vertical using top and down parameter. 
Step8: After that find the disease in the image using left right search function, in which we used transpose function of images and masks.

Step9: Than horizontal scan start and follows same process start after vertical scan

Step10: After that region props coding + skulls coding + compute BC. And then find area rather than length of object or disease.

\section{REFERENCES}

[1] S. M. Bhandarkar and P. Nammalwar, "Segmentation of Multispectral MR images Using Hierarchical SelfOrganizing Map," Proceedings of Computer-Based medical system CBMS 2001.

[2] C. A. Parra, K. Iftekharuddin and R. Kozma, "Automated Brain Tumor segmentation and pattern Recoginition using ANN," Computional Intelligence Robotics and Autonomous Systems, 2003.

[3] Andreas Rimner, Andrei I. Holodny and Fred $\mathrm{H}$. Hochberg,"Perfusion Magnetic Resonance Imaging to Assess Brain Tumor Responses to New Therapies," US neurological disease, 2006.

[4] V. J. Nagalkar and S. S. Asole, "Brain tumor detection using digital image processing based on soft computing," Journal of Signal and Image Processing , Vol. 3,No. 3, pp.-102-105, 2012

[5] Mohammad Shajib Khadem, "MRI Brain image segmentation using graph cuts", Master of Science Thesis in Communication Engineering, Department of Signals and Systems, Chalmers University Of Technology, Goteborg, Sweden, 2010.

[6] Yan Zhu and Hong Yan, "Computerized Tumor Boundary Detection Using a Hopfield Neural Network", IEEE Trans. Medical Imaging, vol. 16, no. 1, pp.55-67 Feb.1997.

[7] Orlando J. Tobias and Rui Seara,'Image Segmentation by Histogram Thresholding Using Fuzzy Sets," IEEE transactions on Image Processing,Vol. 11,NO. 12,PP1457-1465,DEC 2002

[8] Mohamed Lamine Toure, "Advanced Algorithm for Brain Segmentation using Fuzzy to Localize Cancer and Epilepsy Region", International Conference on Electronics and Information Engineering (ICEIE 2010), Vol. no 2.

[9] Minakshi Sharma, Dr. Sourabh Mukharjee, Artificial Neural Network Fuzzy Inference System (ANFIS) For Brain Tumor Detection

[10] D. Jayadevappa, S. Srinivas Kuma2, and D. S. Murty,A Hybrid Segmentation Model based on Watershed and Gradient Vector Flow for the Detection of Brain Tumor, International Journal of Signal Processing, Image Processing and Pattern Recognition Vol. 2, No.3, September 2009
[11] Mukesh Kumar, Kamal K.Mehta, A Texture based Tumor detection and automatic Segmentation using Seeded Region Growing Method

[12] K.Selvanayaki*, Dr. M. Karnan,CAD System for Automatic Detection of Brain Tumor through Magnetic Resonance Image-A Review, K. Selvanayaki et. al. / International Journal of Engineering Science and Technology Vol. 2(10), 2010, 5890-5901

[13] Nobel A.J and BoukerrouiD."Ultrasound Image Segmentation: A survey", IEEE Trans On Medical Imaging ,Vol.25,No.8,pp.987-1010.

[14] Miller P, Astley S. Classification of breast tissue by texture analysis. Image Vision Computer 1992;10:277282.

[15] Koss JE, Newman FD, Johnson TK, Krich DL. Abdominal organ segmentation using texture transform and Hopfield neural network. IEEE Trans Med Imaging 1999;18:640

[16] Manoj K Kowar and Sourabh Yadav, Brain Tumor Detction and Segmentation Using Histogram Thresholding, International Journal of Engineering and Advanced Technology (IJEAT) ISSN: 2249 - 8958, Volume-1, Issue-4, April 201216

[17] Kailash Sinha Shweta Raghuwanshi, An Amend Implementation of Brain Tumor Detection Using Segmentation Based On Artificial Intelligence, International Journal of Digital Application \& Contemporary research Website: www.ijdacr.com (Volume 1, Issue 1, August 2012)

[18] Dina Aboul Dahab, Samy S. A. Ghoniemy, Gamal M. Selim, Automated Brain Tumor Detection and Identification Using Image Processing and Probabilistic Neural Network Techniques

[19] Garima Garg Sonia Juneja, Extract Area of Tumor through MRI using Optimization Technique with Fuzzy C Means, 2012

[20] Meghana Nagori1, Shivaji Mutkule2, Praful Sonarkar, Detection of Brain Tumor by Mining fMRI Images, International Journal of Advanced Research in Computer and Communication Engineering Vol. 2, Issue 4, January 2013

[21] Dr. N. NandhaGopal, Automatic Detection Of Brain Tumor Through Magnetic Resonance Image, International Journal of Advanced Research in Computer and Communication Engineering Vol. 2, Issue 4, April 2013

[22] Vivek Angoth_, A Novel Wavelet Based Image Fusion for Brain Tumor Detection, international Journal of Computer Vision and Signal Processing, 2(1), 1-7(2013)

[23] Kadam D B,et.al, An Artificial Neural Network Approach for Brain Tumor Detection Based on Characteristics of GLCM Texture Features. 